\title{
Evaluation du procédé d'abattage des bovins aux abattoirs de Cotonou-Porto- Novo au sud du Bénin
}

\author{
C.F.A. SALIFOU ${ }^{1}$, A.K.I. YOUSSAO ${ }^{1 *}$, S. SALIFOU ${ }^{1}$, T. M. KPODEKON ${ }^{1}$, \\ P.U. TOUGAN ${ }^{1}$, G.S. AHOUNOU ${ }^{1}$, C. BOCO ${ }^{1}$, S. FAROUGOU ${ }^{1}$, G.A. MENSAH ${ }^{2}$ et \\ A. CLINQUART ${ }^{3}$ \\ ${ }^{1}$ Ecole Polytechnique d'Abomey-Calavi, Département de Production et Santé Animales, \\ 01 BP 2009 Cotonou, Bénin. \\ ${ }^{2}$ Centre de Recherches Agricoles d'Agonkanmey, Institut National des Recherches Agricoles du Bénin, \\ 01 BP 884 Recette Principale, Cotonou 01, Bénin. \\ ${ }^{3}$ Université de Liège, Faculté de Médecine Vétérinaire, Département des Sciences des Denrées Alimentaires, \\ Sart Tilman, 4000 Liège, Belgique. \\ *Auteur correspondant,E-mail: iyoussao@yahoo.fr, issaka.youssao@epac.uac.bj; Tél:0022995285988/ \\ 0022997912074 , Fax : 0022921360199
}

\section{RESUME}

La viande est une denrée alimentaire hautement périssable dont la qualité hygiénique dépend de la contamination pendant les opérations d'abattage et de découpe. L'objectif de l'étude est d'évaluer le procédé d'abattage des bovins aux abattoirs de Cotonou-Porto-Novo au sud-Bénin. L'analyse du procédé d'abattage a été faite sur la base de la règle des cinq $M$. L'hygiène du procédé a été évaluée sur 60 carcasses et au cours de deux périodes (répétition dans le temps). L'analyse du procédé a révélé que les pratiques courantes de production peuvent occasionner la contamination des carcasses par E. coli pathogène, Salmonella enterica, Bacillus cereus, Clostridium botulinum, Clostridium perfringens, Staphylococcus aureus, Listeria monocytogenes, Mycobacterium bovis et Mycobacterium tuberculosis. Les charges microbiennes moyennes par période de prélèvement étaient respectivement de 3,0 $\pm 0,12 \log \mathrm{UFC} / \mathrm{cm}^{2}$ et $5,09 \pm 0,16 \log \mathrm{UFC} / \mathrm{cm}^{2}$ pour la flore aérobie mésophile et $1,2 \pm 0,11 \log \mathrm{UFC} / \mathrm{cm}^{2}$ et $1,73 \pm 0,18 \log \mathrm{UFC} / \mathrm{cm}^{2}$ pour les entérobactériaceae. Un seul échantillon a révélé la présence de Salmonella $s p$. Les charges ont fortement varié selon la période de prélèvement $(\mathrm{P}<0,05)$. Conformément aux critères proposés dans la littérature, l'hygiène du procédé d'abattage peut être considérée satisfaisante durant la première période d'étude et non satisfaisante durant la deuxième période.

(C) 2012 International Formulae Group. All rights reserved

Mots clés: Abattoir, viande, hygiène, microbiologie, procédé, Bénin.

\section{INTRODUCTION}

L'abattoir est tout local approuvé, homologué et ou enregistré par l'autorité compétente, utilisé pour l'abattage et l'habillage d'animaux spécifiés destinés à la consommation humaine. En tant qu'établissement, il doit être situé, conçu et construit de manière à minimiser autant que possible la contamination de la viande et permettre au personnel de travailler dans de 
bonnes conditions d'hygiène (FAO, 2006). Dans de nombreux pays en développement, le manque d'abattoirs adéquats et les méthodes d'abattage insatisfaisantes provoquent des pertes superflues de viande et de sous-produits issus de l'abattage des animaux. De même, des installations inappropriées, des procédures de travail non clairement définies et une main d'œuvre peu compétente, sont les causes essentielles de la mauvaise qualité technologique et hygiénique des viandes produites. Selon les normes requises dans la littérature en matière de construction et d'équipement d'un abattoir, le Bénin dispose d'un complexe d'abattoirs dénommé les abattoirs de Cotonou-Porto-Novo, qui est fonctionnel depuis 1978. En dehors de la vétusté des infrastructures, la main d'œuvre employée et la méthode de travail peu adéquate, font suspecter une mauvaise qualité des viandes issues des abattages. En absence de plan HACCP dans ces abattoirs, il s'avère indispensable de respecter tout au moins les bonnes pratiques d'hygiène et de fabrication conformément aux recommandations de la FAO (FAO, 2006) car l'abattage est une étape critique majeure de l'hygiène des viandes en raison du nombre important d'opportunités de contamination qui peuvent survenir (Dennaïet al., 2001 ; Collobert et al., 2002 ; Vallonton, 2004 ; Merle, 2005 ; Beaubois, 2009). Pour réduire ces opportunités dans les abattoirs de Cotonou-Porto-Novo, l'amélioration du procédé d'abattage est nécessaire sur la base de l'évaluation de l'existant. Cette démarche qualité est en adéquation avec le programme de sécurité sanitaire des aliments dans la politique agricole des Etats membres de l'Union Economique et Monétaire Ouest Africaine (FAO/OMS, 2005). Le Bénin, membre de cette Union, a fixé le «contrôle de la qualité et l'innocuité des aliments» parmi ses objectifs de développement (MAEP, 2001). Pour atteindre ces objectifs, le gouvernement béninois veille à ce que tous les aliments produits ou importés satisfassent aux normes du Codex Alimentarius (2003) soutenues par l'Organisation Mondiale du Commerce. L'objectif de la présente étude est d'évaluer le procédé d'abattage des bovins aux abattoirs de Cotonou-Porto-Novo. De manière spécifique, il s'agit d'établir le diagramme de fabrication de la carcasse, d'analyser le procédé d'abattage et d'évaluer l'hygiène du procédé.

\section{MATERIEL ET METHODES \\ Cadre de l'étude}

Les abattoirs de Cotonou-Porto-Novo sont situés à Akpakpa au point kilométrique 6 sur la route Inter-Etats Bénin-Nigéria, dans le $1^{\mathrm{er}}$ Arrondissement de la Commune de Cotonou. Avec une superficie initiale de 3,5 ha, ils ont fait l'objet d'une occupation anarchique par des populations expropriées. Cette zone d'implantation reconnue marécageuse constitue un obstacle à l'accès aux abattoirs pendant les périodes de pluies. Mis en fonction le $1^{\mathrm{er}}$ mai 1978 , les abattoirs de Cotonou-Porto-Novo sont aujourd'hui situés dans une agglomération dont la densité est de 8.419 habitants au $\mathrm{km}^{2}$ (INSAE, 2010) et constituent une source de nuisance et de pollution permanente pour les populations riveraines. Sur le plan administratif, ils sont sous la tutelle de la Direction de l'Elevage (DE) du Ministère de l'Agriculture, de l'Elevage et de la Pêche (MAEP) et bénéficient d'une gestion autonome.

La collecte des données sur le procédé d'abattage des bovins aux abattoirs de Cotonou-Porto-Novo a été réalisée en deux étapes : une première ayant consisté à analyser le procédé d'abattage afin d'identifier les dangers potentiels liés aux viandes issues de ce procédé et les causes de leur apparition; une deuxième ayant permis d'évaluer l'hygiène du procédé d'abattage.

Les données sur le procédé d'abattage des bovins aux abattoirs de Cotonou-PortoNovo ont été collectées sur des carcasses de bovins en les deux périodes suivantes : de novembre à décembre 2009 pour la première répétition (Période 1); de novembre à décembre 2010 pour la deuxième répétition (Période 2). Le choix des mois de novembre et de décembre est dû au fait que cette période correspond à la saison sèche sur le plan nationale et c'est pendant cette période qu'on 
note une forte concentration des abattages dans les principaux abattoirs du Bénin.

\section{Méthodologie}

L'analyse du procédé d'abattage a été faite suivant la règle des cinq $M$ conformément à la Méthode d'Ishikawa créée par le Professeur Kaoru Ishikawa et utilisée par Valloton (2004); Merle (2005) et Mocho (2005) pour identifier les causes possibles d'apparition des dangers. Cette règle consiste à envisager, à chaque étape de production, la Méthode, la Matière première, la Main d'œuvre, le Milieu et le Matériel comme sources potentielles d'apparition du danger étudié. Seuls les dangers biologiques ont été pris en compte dans la présente étude. Les pratiques d'abattage ont été observées une fois par semaine pendant six semaines, à chaque fois un jour de la semaine différent, pour finalement couvrir tous les jours de la semaine, sauf le dimanche où les abattages ne sont pas organisés. Pour toutes les étapes du procédé, les sources potentielles de contamination ont été inventoriées et des propositions d'amélioration ont été formulées.

Les investigations menées sur l'hygiène du procédé d'abattage ont été réalisées conformément au Règlement (CE) N ${ }^{\circ} 2073 / 2005$ de la Commission Européenne (Commission Européenne, 2005). Pour chaque période, les prélèvements ont été également réalisés un jour par semaine pendant 6 semaines consécutives et conformément à la norme ISO 17604 (ISO, 2003). Le jour de l'échantillonnage a également varié chaque semaine afin que les résultats soient représentatifs de toute la semaine. Quatre prélèvements de $5 \mathrm{~cm}^{2}$ chacun ont été effectués par jour sur cinq demi-carcasses, provenant de cinq animaux différents, alternativement sur une demicarcasse gauche puis sur une demi-carcasse droite. Les sites de prélèvements étaient: le collier, le flanc, le rumsteck et l'épaule. A l'aide d'un emporte-pièce de $2,5 \mathrm{~cm}$ de diamètre, d'une pince et d'une lame à usage unique montée sur un manche de bistouri, les échantillons ont été prélevés juste après l'inspection post-mortem et avant la pesée par la méthode d'excision (méthode destructive) et ont été regroupés aseptiquement par carcasse dans un sachet stomacher stérile préidentifié, refermé et déposé dans une glacière dont la température a été maintenue entre 0 et $4{ }^{\circ} \mathrm{C}$. Les échantillons ont été immédiatement amenés au laboratoire du Département de Production et Santé Animales de l'Ecole Polytechnique d'Abomey-Calavi (EPAC) de l'Université d'Abomey-Calavi (UAC) pour les analyses bactériologiques. Un volume de $100 \mathrm{ml}$ d'eau peptonée préalablement stérilisée a été introduit dans chaque sachet Stomacher contenant la prise d'essai totale de $20 \mathrm{~cm}^{2}$. L'ensemble a été broyé pendant 2 à 3 minutes dans le Stomacher. Le surnageant a été récupéré dans un flacon stérile et a constitué la solution mère à $10^{\circ}$. Les différentes dilutions ont été réalisées à partir de la solution mère et conformément à la norme ISO 6887-2 (ISO, 2004). Les germes recherchés étaient la flore aérobie mésophile (FAM), les entérobactériaceae et les salmonelles qui sont les trois indicateurs de l'hygiène du procédé d'abattage (Règlement N²073/2005 de l'Union Européenne). Les échantillons prélevés dans la matinée ont toujours été ensemencés dans l'après-midi du jour de prélèvement. La FAM a été ensemencée, incubée à $30{ }^{\circ} \mathrm{C}$ et recherchée conformément à la norme ISO 4833 (ISO, 2003); les entérobactériaceae recherchées conformément à la norme ISO 21528-2 (ISO, 2004); les salmonelles recherchées conformément à la norme ISO 6579 (ISO, 2002). Pour chaque germe recherché, les résultats ont été exprimés en termes de log d'unités formant colonie (UFC) par $\mathrm{cm}^{2} \mathrm{de}$ carcasse prélevée. Les logs moyens quotidiens ont été déterminés et affectés à l'une des trois catégories suivantes conformément au

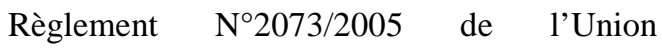
Européenne : satisfaisante (résultat inférieur au minimum), acceptable (résultat entre minimum et maximum) et insatisfaisante (résultat supérieur au maximum). Le minimum en concentration pour la FAM est de 3,5 $\log \mathrm{UFC} / \mathrm{cm}^{2}$ et le maximum est de 5 $\log \mathrm{UFC} / \mathrm{cm}^{2}$. Pour les entérobactériaceae, le minimum en concentration est de 1,5 log 
$\mathrm{UFC} / \mathrm{cm}^{2}$ et le maximum est de 2,5 log $\mathrm{UFC} / \mathrm{cm}^{2}$. Quant aux salmonelles, le résultat est satisfaisant si au plus, 2 analyses sur 50 sont positives et insatisfaisant si plus de 2 analyses sur 50 sont positives.

\section{Analyses statistiques}

Le logiciel Statistical Analysis System (SAS, 1991) a été utilisé pour les analyses statistiques. La moyenne et l'écartype de la FAM et des entérobactériaceae dénombrées ont été calculées par la Procédure Proc Means. Une analyse de variance à deux critères de classification a été réalisée en utilisant la procédure des modèles linéaires généralisées (Proc GLM). Les sources de variations prises en compte étaient le jour de prélèvement et la période de prélèvement. Le test de $\mathrm{F}$ a été utilisé pour déterminer la significativité de chaque facteur de variation et le test $\mathrm{t}$ de Student a été utilisé pour comparer les moyennes deux à deux. Le test de Chi-carré a été utilisé pour comparer les prévalences des salmonelles sur les carcasses par période.

\section{RESULTATS}

\section{Diagramme de fabrication}

Les animaux venant aux abattoirs de

Cotonou-Porto-Novo, provenaient des différents départements du Bénin mais surtout des Départements de l'Alibori et du Borgou au nord-est et de quelques pays voisins tels que le Niger, le Burkina-Faso et le Mali. Ces bovins étaient transportés par des camions, accompagnés par leur propriétaire.

A l'arrivée, les animaux étaient réceptionnés sur le quai de débarquement. Le chef parc procédait à la vérification de l'effectif arrivé par rapport à celui inscrit sur le laissez-passer. Ces bovins subissaient ensuite une inspection sanitaire permettant de séparer les animaux sains des animaux fatigués et les malades. Les bovins sains étaient convoyés au parc de stabulation d'une capacité d'environ 800 têtes où ils séjournaient jusqu'à la veille de leur abattage par un boucher. Ils étaient nourris et suivis quotidiennement.
La veille de l'abattage, après l'inspection ante mortem, les animaux étaient transférés du parc de stabulation au couloir d'amenée vers le local de saignée. Ils y passaient la nuit et étaient sujets à un repos et à une diète hydrique. Les abattages débutaient à 04 heures du matin et prenaient fin au plus tard à 09 heures. Le volume moyen d'abattage était de 70 bovins par jour. Le jour d'abattage, les animaux étaient envoyés un à un dans le local de saignée. Ils étaient contenus grâce au vérin et à l'aide d'un dispositif suspendu à un appareil de levage des animaux par une patte postérieure. Les animaux étaient totalement soulevés du sol puis ramenés à terre. Veine jugulaire orientée vers l'égorgeur, ils étaient égorgés à l'aide d'un couteau à bout courbe bien solide, bien tranchant et bien propre. Remontés quelques minutes plus tard, les animaux étaient lavés par jets d'eau (eau de distribution) après une saignée complète.

L'habillage était réalisé par différentes opérations menées sur l'animal suspendu à un rail sur lequel il cheminait lentement en passant par des postes de travail successifs comme suit :

- la section des membres antérieurs et la mise à nu du sternum dans le local de la saignée se font au premier poste,

- la section de la queue, des pattes postérieures et la mise à nu du train postérieur et de la face ventrale jusqu'au dos étaient réalisées au niveau du $2^{\text {ème }}$ poste de la chaîne. Les animaux étaient enlevés des palonniers et accrochés aux plateaux par les tendons pour la suite des opérations ;

- la fente du sternum par une hachette et le marquage des carcasses par des chiffres apposés au niveau des cuisses de la carcasse et correspondant à la position dans la chaîne d'abattage ( $3{ }^{\text {ème }}$ poste) ;

- l'arrachage du cuir ou dépouille $\left(4^{\text {ème }}\right.$ poste) était réalisé à l'aide de deux chaînes accrochées latéralement à la peau et portant chacune à leur extrémité une masse que l'opérateur saisie et tire pour débarrasser l'animal de sa peau ;

- l'éviscération ( $5^{\text {ème }}$ poste) suivait immédiatement la dépouille; les viscères 
abdominaux étaient enlevés de la cavité abdominale avec tous les soins possibles pour éviter leur éclatement et la souillure de la carcasse. Les viscères abdominaux étaient conduits immédiatement à l'aire de vidange à l'aide d'un chariot. Les viscères thoraciques étaient enlevés et accrochés à la carcasse ;

- la fente médiane de la carcasse $\left(6^{\text {ème }}\right.$ poste) était réalisée à l'aide d'une scie électrique par un ouvrier; la fente commençait à partir de la région de la queue et la face ventrale tournée vers lui, l'opérateur réalisait l'opération jusqu'au niveau de la tête ;

- la section de la tête (6 $6^{\text {ème }}$ poste) était réalisée et après cette opération, chaque demicarcasse était enlevée des plateaux et placée aux crochets ( $7^{\text {éme }}$ poste) avant d'être soumis à l'examen des inspecteurs.

- Après l'inspection post-mortem et l'estampillage des carcasses réalisée à l'aide d'estampille à rouleaux comprenant un cylindre en cuivre où étaient gravées en relief les inscriptions. Les demi-carcasses estampillées étaient pesées grâce à une bascule enregistreuse reliée au rail aérien $\left(8^{\text {ème }}\right.$ poste), avant d'être acheminées soit vers les boucheries, les marchés ou les postes de vente, soit vers la salle de stockage (chambre froide) pour celles devant être conservées pour une date de vente ultérieure. Le ressuage n'était pas pratiqué aux abattoirs de CotonouPorto-Novo.

Les viscères (panse, intestins, foie, rate, poumons et cœur) et les issues (peau et pattes) étaient tous destinés à la consommation. A cet effet, la panse et les intestins sur l'aire de vidange étaient vidés de leur contenu et soumis au premier nettoyage. Ils étaient ensuite ramenés à la boyauderie où ils étaient proprement nettoyés. Les panses étaient maintenues dans l'eau pendant $15 \mathrm{mn}$ pour retrouver leur aspect commercial avant d'être soumis à la vente. Les pattes étaient blanchies dans le but d'enlever les onglons et les poils alors que la peau était brûlée pour enlever les poils afin d'obtenir une sorte de viande communément appelée «kpaman». La tête quant à elle, était disséquée pour mettre à nue les ganglions lymphatiques, rétropharyngiens et sous glossiens afin de faciliter leur examen par le vétérinaire lors de l'inspection. Les abats rouges, à l'exception du cœur et des poumons accrochés à la carcasse, le foie et la rate étaient dépourvus de leurs membranes puis installés sur la paillasse et marqués par le numéro de leur carcasse respective. Ils étaient ensuite présentés à l'examen de salubrité avec la tête et les intestins. Tout le cinquième quartier (tête, foie, rate, cœur, poumons et boyaux) était par la suite ramené à la boyauderie et mis en vente. Le procédé d'abattage de chaque bovin a duré au plus une heure de temps et était composé des 18 étapes suivantes: la réception des animaux; l'inspection ante mortem; l'amenée; la contention; la saignée; l'égouttage; le douchage; la section des membres antérieurs et la mise à nu du sternum; la section de la queue, des pattes postérieures et la mise à nu du train postérieur; la fente du sternum; l'arrachage du cuir et le marquage de la carcasse; l'éviscération; la fente de la carcasse en deux demis carcasses avec à l'intérieur d'une des demis carcasses les rognons; la section de la tête; l'inspection post mortem; l'estampillage ; la pesée ; le stockage.

\section{Analyse du procédé d'abattage}

L'analyse du procédé d'abattage a permis de mettre en évidence des pratiques occasionnant une contamination des carcasses et d'identifier des dangers potentiels associés à la viande de même que leurs causes d'apparition et les mesures préventives associées.

\section{Matières premières}

L'animal était lui-même une source de contamination. La peau était souvent salie par diverses souillures, la boue ou les matières fécales. Le parc de stabulation des abattoirs de Cotonou-Porto-Novo étant boueux, plus de $50 \%$ des animaux déjà partiellement souillés avant leur arrivé à l'abattoir l'étaient davantage avant d'entrer dans le couloir d'abattage. Le contact des carcasses entre elles, le contact du contenu des viscères avec les carcasses et le contact du cuir avec la 
carcasse étaient régulièrement observés. D'autres sources de contamination pouvaient être observées à travers les organes respiratoires ou la mamelle dans le cas d'une mammite. Les bâtiments étaient nettoyés avec de l'eau de puits de qualité hygiénique non connue. La surface des carcasses pouvait ainsi être contaminée par des micro-organismes pathogènes comme E. coli pathogène, Clostridium botulinum, Listeria monocytogenes et Mycobacterium bovis. Pour prévenir ou diminuer la contamination de surface des carcasses,-i- rien que des animaux propres devaient être introduits dans la chaîne d'abattage, -ii- un espace suffisant devait être observé entre les carcasses dans la chaîne d'abattage afin d'éviter tout contact du cuir et du contenu des viscères avec les carcasses, et iii- de l'eau potable, de préférence de l'eau de distribution devait être utilisée. Pour prévenir l'apport de germes par les contenus digestifs, la ligature de l'œsophage devait être réalisée pour éviter la contamination de la carcasse par l'écoulement de leur contenu. De la même façon, une attention particulière devait être faite afin de ne pas percer les réservoirs digestifs lors de l'éviscération. La contamination par la sphère uro-génitale pouvait être réduite de la même façon pour les réservoirs digestifs en évitant les perforations. Enfin, l'ensemble trachéo-pulmonaire devait être enlevé d'un bloc et sans perforation.

\section{Matériel}

Les matériels utilisés lors de la préparation de la carcasse peuvent être à l'origine de la contamination de la viande lorsqu'ils étaient souillés. Aux abattoirs de Cotonou-Porto-Novo, le même couteau était utilisé pour une même étape du diagramme de fabrication sans être rincé ou nettoyé entre deux carcasses et ce, du début jusqu'à la fin des abattages journaliers. Exceptionnellement, si une contamination visuelle survenait, l'opérateur à son poste, rinçait l'outil à l'aide d'eau de distribution fournie par l'intermédiaire des raccords reliés à des points d'eau installés à des endroits précis dans le hall d'abattage. A la fin des abattages, les couteaux utilisés étaient souvent rincés à l'eau et n'étaient pas désinfectés à l'eau chaude ou par d'autres techniques de désinfection. Les anfractuosités observées au niveau de la scie, de la hachette servant à fendre le sternum, des crochets et du dispositif de pesée utilisés dans cet abattoir hébergent des résidus de diverses matières et sans doute des germes difficilement accessibles au nettoyage. En dehors de la scie qui était rincée en fin de journée, la hachette, les crochets et le dispositif de pesée n'étaient tout au moins pas rincés. Les ouvriers étaient en tenues non spécifiques pour l'abattage et ces tenues n'étaient pas lavées ou changées quotidiennement. Elles étaient le plus souvent souillées par des matières fécales, du sang et divers résidus. De même, les bottes ou les chaussures utilisées par les ouvriers et les apprentis n'étaient régulièrement pas lavées. Les véhicules de transport des animaux après le déchargement étaient juste balayés ou rincés à l'eau, malgré la présence de déjections et de jetages. La mauvaise hygiène du matériel pouvait favoriser la contamination de la carcasse par des germes pathogènes comme, Salmonella enterica, Bacillus cereus, Clostridium botulinum, Clostridium perfringens, Listeria monocytogenes, etc. Pour les mesures préventives, un accent particulier devait être mis globalement sur le nettoyage et la désinfection. La stérilisation ou un lavage à l'eau chaude des couteaux, de la scie et de la hachette après chaque carcasse devait être systématique. L'ensemble de l'installation d'abattage devait être quotidiennement désinfecté. Les tabliers imperméables portés par quelques uns devaient être rincés régulièrement à l'eau. Les transporteurs d'animaux de boucherie devaient utiliser des bétaillères ou des véhicules de transport construits afin de pouvoir débarquer les animaux facilement sans risque de blessure et de souillure. Ainsi, ces véhicules devaient être conçus de manière à pouvoir être nettoyés et désinfectés facilement.

\section{Milieu}

Le milieu concernait les locaux, l'air ambiant et l'environnement de l'abattoir. Les abattoirs de Cotonou-Porto-Novo étaient 
implantés dans une zone marécageuse où l'eau stagnait pendant la saison des pluies. Ces marécages étaient des dépotoirs où s'accumulaient du contenu du tractus digestif, des saisies et des déchets d'abattage. L'eau du marécage favorisait la décomposition de ces déchets organiques avec pour corollaire une multiplication des germes pathogènes et un dégagement d'odeurs nauséabondes, d'où la pollution de l'air qui circulait dans les environs. Les abattoirs de Cotonou-PortoNovo, abritaient des mammifères rongeurs, des margouillats, des insectes, des araignées, etc. Le sol ou plancher n'a jamais été renouvelé depuis la création de l'abattoir. Aujourd'hui, ce plancher est rugueux et creusé par endroit. Ces creux contenaient des résidus d'eaux usées favorables à la prolifération des micro-organismes. Néanmoins, l'existence d'une pente suffisante permettait le drainage des eaux usées vers les canaux. La température dans la seule chambre fonctionnelle variait très souvent de +2 à +8 ${ }^{\circ} \mathrm{C}$ au maximum $\left(8^{0} \mathrm{C}\right.$ pendant les ouvertures $)$ et ne permettait pas une conservation de longue durée. Aucune des quatre autres chambres froides restantes n'était fonctionnelle et servaient de locaux de stockage de certains matériels de travail. L'air véhiculait diverses bactéries notamment $E$. coli pathogène, Salmonella enterica, Staphylococcus aureus, Clostridium botulinium, Clostridium perfringens, Bacillus cereus, Listeria monocytogenes, etc.

L'assainissement du milieu exigeait un drainage des eaux du marécage, une meilleure gestion des déchets, la séparation rigoureuse des secteurs propres et souillés. Le déplacement du personnel devait se faire uniquement du secteur propre vers le secteur souillé. A chaque étape du diagramme de fabrication, devait correspondre un agent de poste. Le sol, les murs, le plafond et les portes devaient être facilement lavables et désinfectés régulièrement en absence de carcasse. Les fissures, les trous, la rouille, etc. devaient être évités et le flux d'air devait être orienté du secteur propre vers le secteur souillé. En outre, les chambres froides devaient redevenir fonctionnelles.

\section{Méthodes}

Même si des contaminations étaient inévitables au cours des différentes étapes du processus d'abattage, il était néanmoins possible de les limiter en respectant certaines méthodes de travail. Aux abattoirs de Cotonou-Porto-Novo, les causes favorisant la contamination des carcasses étaient les suivantes : le stress des animaux avant l'abattage; la régurgitation dans la plaie de saignée; le passage du contenu stomacal dans les poumons; la propagation des souillures du cuir dans la plaie de la saignée lors du douchage; le contact entre les carcasses. Par conséquent, la formation de tout le personnel au bien-être des animaux et aux bonnes pratiques à appliquer en industrie de la viande était indispensable. Pour chaque poste, des fiches de poste devaient être élaborées en tenant compte des contaminations bactériennes potentielles et de la façon à optimiser le travail tout en réduisant le risque bactérien. La section simultanée de l'œsophage, de la trachée et de la veine jugulaire devait être évitée à l'étape de saignée aux abattoirs de Cotonou-Porto-Novo. Par ailleurs, le croisement des carcasses devait être évité sur la chaîne d'abattage. En cas de souillure d'une zone de la carcasse par exemple, les matières fécales devaient être enlevées manuellement et superficiellement avec un couteau la zone souillée. En cas de défaillances dans l'application des bonnes pratiques d'abattage, la probabilité que la carcasse ait été contaminée par E. coli pathogène, Salmonella enterica, Bacillus cereus, Clostridium botulinum, Clostridium perfringens et Listeria monocytogenes était élevée.

\section{Main d'œuvre}

La main d'œuvre a concerné toutes les personnes impliquées dans la chaîne d'abattage aussi bien en amont pour le déchargement des animaux et leur amenée dans le couloir d'abattage qu'en aval pour l'expédition des carcasses et la découpe en quartiers. N'importe quel opérateur pouvait être porteur de germes pathogènes au niveau intestinal, cutané ou bucco-pharyngé. Une 
mauvaise hygiène du personnel a été observée. En effet, certains ouvriers se curaient les dents pendant les opérations d'abattage et d'autres portaient la même tenue plusieurs jours consécutifs. Par conséquent, pour observer une bonne hygiène au niveau de la main d'œuvre, les actions suivantes étaient nécessaires :

- prévoir plus de locaux sanitaires pour le personnel et les maintenir dans un bon état de propreté ;

- installer des robinets à commande non manuelle, du savon, du désinfectant, de même qu'un dispositif hygiénique de séchage des mains ;

- former les acteurs à l'hygiène du personnel au respect des fiches de poste;

- mettre à la disposition des opérateurs des vêtements de couleur claire (uniforme) et des chaussures étanches comme des paires de bottes réservées spécialement à la zone d'abattage;

- changer quotidiennement les tenues de travail, laver et désinfecter avant ou après abattage les chaussures de travail ;

- nettoyer et désinfecter les mains régulièrement après toute contamination et porter des gants en cas de plaie sur les mains ; bien que l'idéal devait être le port des gants de façon systématique pendant les diverses opérations;

- contrôler la santé du personnel à travers un bilan de santé annuel.

En somme, la main d'œuvre a été parmi les 5 $\mathrm{M}$, le domaine le plus incontrôlable.

\section{Hygiène du procédé d'abattage}

La charge moyenne en FAM obtenue pour les cinq carcasses prélevées quotidiennement a varié du lundi au samedi, de 1,96 à 3,64 $\log \mathrm{UFC} / \mathrm{cm}^{2}$ sans aucune différence significative pour la période 1 et de 3,98 à $6,17 \log \mathrm{UFC} / \mathrm{cm}^{2}$ pour la période 2 (Tableau 1). Pendant les trois premiers jours de la semaine, la charge en FAM n'a pas été différente pour les deux périodes. Par contre, du jeudi au samedi, la charge en FAM a été plus importante pour la période 2 par rapport à celle de la période $1 \quad(\mathrm{P}<0,01)$ et a varié significativement par rapport aux trois premiers jours de la semaine. Pour toute la période de l'étude, la valeur moyenne de la charge microbienne de la période $2(5,09 \pm$ $0,16 \log \mathrm{UFC} / \mathrm{cm}^{2}$ ) a été significativement 1,7 fois plus élevée $(\mathrm{P}<0,01)$ que celle de la Période $1\left(3,0 \pm 0,12 \log \mathrm{UFC} / \mathrm{cm}^{2}\right)$ pour la FAM. Sur le plan qualitatif, la charge en FAM était globalement satisfaisante pendant la première période et non satisfaisante pendant la deuxième période.

Concernant les entérobactériaceae, la charge microbienne a été dans l'ensemble satisfaisante pour la première période $(1,2 \pm$ $\left.0,11 \log \mathrm{UFC} / \mathrm{cm}^{2}\right)$ et acceptable pour la deuxième $\left(1,73 \pm 0,18 \mathrm{log} \mathrm{UFC} / \mathrm{cm}^{2}\right)$ (Tableau 2 ). Toutefois, aucune différence significative n'a été observée entre les charges moyennes des deux périodes $(\mathrm{P}>0,05)$. Les charges en entérobactériaceae étaient acceptables les mercredis et les samedis pour la période 1 et non satisfaisantes pour la période 2. Cette différence était en correspondance avec les charges moyennes en entérobactériaceae qui étaient fortement élevées dans la période 2 comparativement à celles de la période 1 $(\mathrm{P}<0,01)$. L'effet jour n'a pas été observée pour les charges en entérobactériaceae pendant la période 1 , alors que dans la période 2, les charges supérieures à la limite tolérable ont été obtenues les mercredis et les samedis aux abattoirs de Cotonou-Porto-Novo $(\mathrm{P}<0,05)$.

Tous les échantillons prélevés durant la période 1 étaient exempts de Salmonella. Un seul échantillon sur les 30 analysés dans la période 2 a révélé la présence de microorganisme du genre Salmonella. Ainsi, pour les 60 échantillons analysés, la présence de salmonelle a été détectée dans un seul échantillon. Par conséquent, la qualité hygiénique du procédé d'abattage était satisfaisante aux abattoirs de Cotonou-PortoNovo. 
Tableau 1: Dénombrement de la flore aérobie mésophile des carcasses de bovins abattus aux abattoirs de Cotonou-Porto-Novo.

\begin{tabular}{|c|c|c|c|c|}
\hline \multirow{2}{*}{$\begin{array}{l}\text { Jours de } \\
\text { prélèvement }\end{array}$} & \multicolumn{2}{|c|}{ Période 1} & \multicolumn{2}{|c|}{ Période 2} \\
\hline & $\begin{array}{c}\text { Moyenne } \pm \text { ET } \\
\left(\log \mathrm{UFC} \mathrm{cm}^{2}\right)\end{array}$ & Interprétation* & $\begin{array}{c}\text { Moyenne } \pm \text { ET } \\
\left(\log \mathrm{UFC} / \mathrm{cm}^{2}\right)\end{array}$ & Interprétation* \\
\hline Lundi & $3,58 \pm 0,83 a$ & Acceptable & $4,33 \pm 1,21 \mathrm{a}$ & Acceptable \\
\hline Mardi & $2,44 \pm 0,21 a$ & Satisfaisant & $3,98 \pm 0,87 a$ & Acceptable \\
\hline Mercredi & $3,45 \pm 0,47 \mathrm{a}$ & Satisfaisant & $4,65 \pm 1,42 \mathrm{a}$ & Acceptable \\
\hline Jeudi & $3,01 \pm 0,79 a$ & Satisfaisant & $5,56 \pm 2,10 b$ & Non satisfaisant \\
\hline Vendredi & $1,96 \pm 0,67 a$ & Satisfaisant & $6,17 \pm 2,57 b$ & Non satisfaisant \\
\hline Samedi & $3,64 \pm 0,05 a$ & Acceptable & $5,87 \pm 1,64 b$ & Non satisfaisant \\
\hline Total & $3,01 \pm 0,68 a$ & Satisfaisant & $5,09 \pm 0,89 b$ & Non satisfaisant \\
\hline
\end{tabular}

ET : Ecart-type, * Conformément aux normes prévues par l'Union Européenne (2005), le minimum en concentration est de 3,5 $\log \mathrm{UFC} / \mathrm{cm}^{2}$ et le maximum $5 \log \mathrm{UFC} / \mathrm{cm}^{2}$ pour la Flore aérobie mésophile (FAM). Les moyennes de la même ligne, suivies des lettres différentes, diffèrent significativement au seuil de $5 \%$.

Tableau 2: Dénombrement des Entérobactériaceae des carcasses de bovins abattus aux abattoirs de Cotonou-Porto-Novo.

\begin{tabular}{|c|c|c|c|c|}
\hline \multirow{2}{*}{$\begin{array}{l}\text { Jour de } \\
\text { prélèvement }\end{array}$} & \multicolumn{2}{|c|}{ Période 1} & \multicolumn{2}{|c|}{ Période 2} \\
\hline & $\begin{array}{r}\text { Moyenne } \pm \text { ET } \\
\left(\log \mathrm{UFC} / \mathrm{cm}^{2}\right)\end{array}$ & Interprétation* & $\begin{array}{r}\text { Moyenne } \pm \text { ET } \\
\left(\log U F C / \mathrm{cm}^{2}\right)\end{array}$ & Interprétation* \\
\hline Lundi & $0,49 \pm 0,45 a$ & Satisfaisant & $0,55 \pm 0,88 a$ & Satisfaisant \\
\hline Mardi & $1,06 \pm 1,19 a$ & Satisfaisant & $1,11 \pm 1,08 \mathrm{a}$ & Satisfaisant \\
\hline Mercredi & $1,69 \pm 1,07 \mathrm{a}$ & Acceptable & $2,81 \pm 0,56 b$ & Non satisfaisant \\
\hline Jeudi & $0,97 \pm 1,04 \mathrm{a}$ & Satisfaisant & $1,11 \pm 0,98 \mathrm{a}$ & Satisfaisant \\
\hline Vendredi & $1,10 \pm 0,71 \mathrm{a}$ & Satisfaisant & $1,61 \pm 0,69 a$ & Acceptable \\
\hline Samedi & $2,06 \pm 0,56 a$ & Acceptable & $3,17 \pm 0,98 b$ & Non satisfaisant \\
\hline Total & $1,2 \pm 0,61 \mathrm{a}$ & Satisfaisant & $1,73 \pm 1,04 a$ & Acceptable \\
\hline
\end{tabular}

\section{DISCUSSION}

Le diagramme du procédé d'abattage des bovins montre que l'abattage pratiqué est de type halal. L'absence de l'étape d'étourdissement présente des risques aussi bien pour l'opérateur que pour l'animal (risque de stress). Une contention adaptée doit permettre d'y remédier. Par rapport aux pratiques d'abattage recommandées (FAO, 2006), aux abattoirs de Cotonou-Porto-Novo, il manque dans le diagramme de fabrication des carcasses de bovins, l'étourdissement, la ligature de l'œsophage et de l'anus, l'ablation du museau, l'aspiration de la moelle épinière et le prélèvement pour l'ESB (Encéphalopathie Spongiforme Bovine) réalisé dans les pays européens. La plupart de ces étapes qui ne figurent pas dans le diagramme de fabrication des carcasses aux abattoirs de Cotonou-Porto-Novo, sont des points importants pour la maîtrise du risque lié à l'agent de l'ESB. D'ailleurs, dans les pays de l'Union Européenne, ces étapes ont été ajoutées au diagramme classique depuis l'apparition de la maladie « de la vache folle » (FAO, 2006). En dehors des abattoirs de 
Cotonou-Porto-Novo, les autres communes du Bénin ne disposent que de tueries dans lesquelles une diversité importante des procédés d'abattage existe. Dans la Commune de Banikoara, trois diagrammes différents de fabrication des carcasses ont été récapitulés (Dognon, 2010). Par rapport au diagramme de Cotonou-Porto-Novo, une absence totale de l'inspection ante mortem a été observée et cela se justifie par le fait que la quasi-totalité des viandes consommées dans la commune soient des viandes foraines provenant des animaux malades ou accidentés et abattus hors des tueries et des aires d'abattage où se font habituellement les inspections sanitaires.

L'analyse du procédé d'abattage révèle également que les viandes bovines issues des abattoirs de Cotonou-Porto-Novo peuvent être associées à de nombreux dangers biologiques pour l'homme tels que E. coli pathogène, Salmonella enterica, Bacillus cereus, Clostridium botulinum, Clostridium perfringens, Staphylococcus aureus, Listeria monocytogenes, Mycobacterium bovis, Mycobacterium tuberculosis et Bacillus anthracis. La plupart de ces dangers biologiques sont récapitulés par Merle (2005) lors du bilan de la mise en œuvre de la démarche HACCP dans les abattoirs en France et par Fosse et al. (2006) lors de l'étude sur la typologie des dangers transmis à l'homme par la consommation des viandes. Parmi ces dangers, ceux qui sont les plus fréquemment incriminés dans des cas de toxiinfection alimentaire et dont les signes cliniques induits et les plus graves sont Salmonella enterica, Listeria monocytogenes, Staphylococcus aureus, Clostridium perfringens et Clostridium botulinum (Vaillant et al., 2004).

Dans la plupart des cas, les dangers bactériens n'induisent pas de lésions spécifiques justifiant une saisie. Parmi les dangers répertoriés aux abattoirs de CotonouPorto-Novo, seul Mycobacterium bovis peut être détecté lors de l'inspection post-mortem si des lésions sont visibles. Pour les autres, aucune mesure préventive n'est prise pour empêcher leur apparition liée à des non- conformités relatives à la construction, la configuration et l'équipement de l'abattoir et à l'hygiène du personnel. En ce qui concerne la limitation de la contamination des carcasses par des bactéries, un effort louable est fait en matière d'hygiène lors de l'abattage comme suit : égouttage complet du sang; dépouille satisfaisante ; pas de contact entre les abats ; les viscères et les carcasses; les carcasses n'entrant pas en contact avec le sol, le mur et le poste de travail. Gill et al. (1998) rapportent que la contamination superficielle des carcasses varie en quantité et en qualité selon la méthode de dépouille utilisée et qu'elle est peu fonction de l'ouvrier. Les nonconformités majeures observées sont l'absence de changement des couteaux ou l'absence de stérilisation des couteaux, des scies ou tout autre matériel utilisé, ainsi que l'absence de lavage régulier des mains des opérateurs après chaque opération.

Le ressuage n'est pas pratiqué aux abattoirs de Cotonou-Porto-Novo car les carcasses sont vendues immédiatement après l'inspection post mortem. Les carcasses qui ne sont pas destinées immédiatement à la vente sont conservées dans des chambres froides où le refroidissement ne permet pas d'atteindre une température à cœur conforme $\left(\leq+7{ }^{\circ} \mathrm{C}\right.$ le plus rapidement possible après l'abattage et le maintien de cette température pendant le stockage et la distribution). Tout le procédé d'abattage doit être revu compte tenu des mesures préventives qui ne sont pas prises pour presque toutes les opérations alors qu'elles sont indispensables pour garantir la sécurité sanitaire du produit fini. Au préalable, le personnel doit être formé aux bonnes pratiques pour l'industrie des viandes.

Quant à l'hygiène du procédé d'abattage, les travaux de la première période révèlent que l'hygiène du procédé d'abattage aux abattoirs de Cotonou-Porto-Novo est satisfaisante tandis que les travaux de la deuxième période indiquent le contraire. La charge quotidienne moyenne en FAM des carcasses échantillonnées varie du lundi au samedi. Sur le plan qualitatif, la charge en FAM est satisfaisante pendant la première 
période et non conforme pour la deuxième période. La charge en entérobactériaceae est dans l'ensemble satisfaisante pour la première période et acceptable pour la deuxième. Cette variabilité de la charge en microorganismes indique que l'hygiène du procédé d'abattage aux abattoirs de Cotonou-Porto-Novo n'est pas constante dans le temps. Toutefois, la température ambiante, la saison et l'état de propreté des animaux ou de l'environnement peuvent être à la base de cette observation. La forte charge en FAM observée dans la période 2 indique d'une part une hygiène générale défectueuse des carcasses impliquant leur non-conformité et d'autre part, l'inefficacité des mesures hygiéniques. $\mathrm{Au}$ regard du diagnostic hygiénique établi, le nombre de germes obtenus n'est pas très alarmants et le risque aurait été grand si la cuisson des viandes en Afrique et particulièrement au Bénin n'était généralement pas complète. En effet, les viandes sont habituellement bouillies et frites ou braisées jusqu'à une cuisson à cœur complète au Bénin.

La variation de la charge en fonction du jour du prélèvement prouve une instabilité dans la méthode de travail. La présence de salmonelles est détectée dans un seul échantillon. Des prévalences similaires sont rapportées par Philippes et al. (2001) qui ont détecté des salmonelles sur $0,2 \%$ des carcasses échantillonnés et sur $0,1 \%$ de viande de bœuf désossées en Australie. Les charges obtenues dans la présente étude peuvent être moins importantes si les animaux ne sont pas douchés avant l'habillage. En effet, le niveau de contamination superficielle de la carcasse est influencé par l'état de propreté visuelle de l'animal avant l'abattage ou avant l'habillage. Les travaux de McEvoy et al. (2000) rapportent l'existence d'une différence de contamination pour la FAM superficielle des carcasses entre les animaux peu sales et les animaux très sales. Par contre, aucune différence significative $(p>0,05)$ n'existe entre les animaux peu sales et moyennement sales, ni entre les animaux moyennement sales et très sales (McEvoy, 2000). Des notes de propreté peuvent être appliquées aux animaux qui entrent aux abattoirs de Cotonou-PortoNovo afin de prendre des mesures comme par exemple abattre les animaux les plus sales après avoir abattu les plus propres si certains niveaux sont dépassés.

Au Bénin et dans la plupart des pays de l'Afrique subsaharienne, peu d'études sont réalisées sur les contaminants des carcasses lors du processus d'abattage. Dans la Commune de Banikoara, la contamination moyenne est de 7,61 $\mathrm{log} \mathrm{UFC} / \mathrm{cm}^{2}$ pour la FAM et $4,1 \log \mathrm{UFC} / \mathrm{cm}^{2}$ pour les entérobactériaceae (Dognon, 2010). La présence de salmonelles a été détectée sur $72 \%$ de l'ensemble des carcasses analysées (Dognon, 2010). De ce fait, les résultats de contaminations microbiologiques des carcasses de bovins dans cette tuerie sont largement au-dessus des limites généralement fixées. Les charges microbiennes enregistrées sur les carcasses de bovins aux abattoirs de Cotonou-Porto-Novo sont largement en dessous de celles enregistrées à Banikoara. Un dénombrement semblable à celui effectué dans la présente étude est rapporté par Collobert et al. (2002) sur 233 carcasses de bovins abattus dans quatre abattoirs du Calvados où la contamination moyenne est de $3,78 \log U F C / \mathrm{cm}^{2}$ pour la FAM et $1,42 \mathrm{log}$ $\mathrm{UFC} / \mathrm{cm}^{2}$ pour les entérobactériaceae. El Okki et al. (2005) ont dénombré 5,34 $\log \mathrm{UFC} / \mathrm{cm}^{2}$ de carcasse pour la FAM à l'abattoir municipal de Constantine en Algérie. Sur une chaîne d'abattage à Toulouse, les niveaux de contamination annuels varient de 0 à 2,3 log $\mathrm{UFC} / \mathrm{cm}^{2}$ pour les entérobactériaceae et de 3,8 à $4,8 \log \mathrm{UFC} / \mathrm{cm}^{2}$ pour la FAMT (Vallonton, 2004). Toutefois, la variation au cours du temps des charges dénombrées sur les carcasses ne permet pas de statuer sur l'hygiène du procédé d'abattage des bovins aux abattoirs de Cotonou-Porto-Novo et de faire une comparaison valable avec les abattoirs d'autres pays. Il faille songer à l'amélioration de l'hygiène de l'abattage et un réexamen des contrôles de procédé. 


\section{Conclusion}

L'évaluation du procédé d'abattage des bovins aux abattoirs de Cotonou-PortoNovo révèle que l'hygiène du procédé d'abattage est instable dans le temps et que les carcasses sont sujettes à des contaminations diverses liées aux pratiques d'abattage. Les principaux agents pathogènes potentiels de contamination sont E. coli pathogène, Salmonella enterica, Bacillus cereus, Clostridium botulinum, Clostridium perfringens, Campylobacter jejuni, Staphylococcus aureus, Streptocoques fécaux, Listeria monocytogenes, Mycobacterium bovis, Mycobacterium tuberculosis. Quant à la qualité microbiologique, la charge en FAM apparaît inconstante selon la période de prélèvement. La charge en entérobactériaceae est dans l'ensemble satisfaisante à acceptable. Cette variabilité de la charge en microorganismes indique que l'hygiène du procédé d'abattage dans les abattoirs de Cotonou-Porto-Novo n'est pas constante dans le temps. La variation de la charge en fonction du jour de prélèvement prouve une instabilité dans la méthode de travail aux abattoirs de Cotonou-Porto-Novo. Par conséquent, la qualité hygiénique du procédé d'abattage est insatisfaisante aux abattoirs de CotonouPorto-Novo. A l'instar des abattoirs de Cotonou-Porto-Novo, l'amélioration de l'hygiène générale des aires d'abattage ou tueries du Bénin s'avère indispensable et doit être possible grâce à la mise en place de contraintes auprès des éleveurs pour un acheminement d'animaux propres et la réadaptation des locaux des abattoirs conformé mentaux textes règlementaires adopté par le Bénin. Le personnel des abattoirs doit être formé aux bonnes pratiques de production et d'hygiène dans une industrie de viande. Un plan HACCP urge d'être mis en place avec l'utilisation des indicateurs bactériologiques en plus des indicateurs lésionnels habituellement utilisés lors des opérations d'inspection. Aussi, une motivation des responsables et des opérateurs doit permettre d'améliorer ces indicateurs et d'objectiver l'intérêt de leurs efforts.

\section{REMERCIEMENTS}

Les auteurs remercient la coopération universitaire au développement (cud) pour son appui à la réalisation de ces travaux a travers le troisième programme quinquennal (p3) de la coopération universitaire institutionnelle (cui) du conseil interuniversitaire de la communauté française de Belgique (ciuf). Les remerciements sont également adresses aux responsables de l'activité uac01.

\section{REFERENCES}

Beaubois P. 2009. Qualité microbiologique de la viande bovine: maîtrise sanitaire des produits carnés, exemple chez SOCOPA. Viandes Prod. Carnés, 26(4): 123-126.

Codex Alimentarius. 2003. Recommended International Code of Practice - General Principles of Food Hygiene, CAC/RCP1-1969, Rév., 4, p. 31.

Collobert J, Dorey F, Dieuleveux V. 2002. Qualité bactériologique de surface de carcasses de bovins. Sci. Alim., 22: 327334.

Commission Européenne. 2005. Règlement (CE) $n^{\circ} 2073 / 2005$ de la Commission du 15 novembre 2005 concernant les critères microbiologiques applicables aux denrées alimentaires. J. Officiel Union Europ.

Dennaï N, Kharrattib B, El YachiouimA. 2001. Appréciation de la qualité microbiologique des carcasses de bovins fraîchement abattus. Ann. Méd. Vét., 145: 270-274.

Dognon R. 2010. Evaluation de la qualité hygiénique des carcasses de bovins fraîchement abattus en milieu rural : cas de la commune de Banikoara. Mémoire de Master en Normes, Contrôle de Qualité et Technologie Alimentaire, Université d'Abomey-Calavi, Bénin, p. 71.

El Okki S, El Groud R, Kenana H, Quessy S. 2005. Évaluation de la contamination superficielle des carcasses bovines et ovines provenant de l'abattoir municipal de Constantine en Algérie. Can. Vet. J., 200: 638-640. 
FAO/OMS. 2005. Conférence régionale sur la sécurité sanitaire des aliments pour l'Afrique, du 3 au 6 octobre 2005, Harare, Zimbabwe. Consulté le 12 mars 2010 sur le site web ftp://ftp.fao.org/docrep/fao/meeting/009/ ae723f.pdf

FAO. 2006. Production et sante animales : Bonnes pratiques pour l'industrie de viande de la viande. Consulté le 12 mars 2010 sur le site web ftp://ftp.fao.org/docrep/fao/009/y5454f/y 5454f.pdf

Fosse J, Cappelier J-M, Laroche M, Fradin N, Giraudet K, Magras C. 2006. Viandes bovines: une analyse des dangers biologiques pour le consommateur appliquée à l'abattoir. Renc. Rech. Ruminants, 13: 411-414.

Gill CO, Mcjinnis JC, Bryan J. 1998. Microbial contamination of meat during the skinning of beef carcass hindquarters at three slaughtering plants. Int. J. Food Microbiol., 42: 175-184.

INSAE (Institut National de la Statistique et de l'Analyse Economique). 2010. Institut National de la Statistique et de l'Analyse Economique, Rapport Annuel d'Activités, Cotonou, République du Bénin, p. 231.

ISO 6579. 2002. Méthode horizontale pour la recherche de Salmonella spp. V08-013, $1-26$.

ISO 17604. 2003. Microbiologie des aliments. Prélèvement d'échantillon sur des carcasses en vue de leur analyse microbiologique. p. 15.

ISO 4833. 2003. Méthode horizontale pour le dénombrement des micro-organismes. V08-011, 1-9.

ISO 6887-2. 2004. Préparation des échantillons, de la suspension mère et des dilutions décimales en vue de l'examen microbiologique. V08-010-2, p. 16.
ISO 21528-2, 2004. Méthode horizontale pour la recherche et le dénombrement des entérobactériaceae. V08-039-2, p. 12.

MAEP (Ministère de l'Agriculture, de l'Elevage et de la Pêche) 2001. Déclaration de la Politique de Développement Rural (DPDR), Cotonou, République du Bénin, p. 241.

Mcevoy JM, Doherty AM, Finnerty M, Sheridan JJ, Mcguire L, Blair IS, Mcdowell DA, Harrington D. 2000. The relationship between hidecleanliness and bacterial numbers on beef carcasses at a commercial abattoir. Letters in Applied Microbiology, 30: 390-395.

Merle EM, 2005. Application de la méthode HACCP en abattoir : bilan de deux années de mise en œuvre. Thèse de Docteur Vétérinaire, ENVT, Toulouse, France, p. 101.

Mocho J-P. 2005. Évaluation de l'hygiène sur une chaîne d'abattage ovin à l'aide d'examens bactériologiques de surface des carcasses. Thèse de Docteur Vétérinaire, ENVT, Toulouse, France, p. 57.

Philippes D, Sumner S, Alexander JF, Dutton KM. 2001. La qualité microbiologique de la viande bovine australienne. Prot. Aliment. J., 64(5): 692-696.

SAS, SAS/STAT. 1991. User's guide (version $6,4^{\text {th }}$ edn.). Inst. Inc., Cary, NC, USA.

Vaillant V, De Valk H, Baron E. 2004. Morbidité et Mortalité Dues aux Maladies Infectieuses d'Origine Alimentaire en France ( $1^{\text {ère }}$ edn). Institut de Veille Sanitaire: Saint-Maurice; 192.

Vallonton FM. 2004. Evaluation de l'hygiène sur une chaîne d'abattage bovin à l'aide d'examens bactériologiques de surface, Thèse de Médecine Vétérinaire, ENVT, Toulouse, p. 74. 